\title{
Modeling of Transient Two Dimensional Flow in Saturated-Unsaturated Porous Media
}

\author{
Hassan Lemacha \\ Laboratory of Applied Geology, Geomatics and Environment, Department of \\ Geology, Faculty of Sciences Ben M'sik, Hassan II-Casablanca University, \\ B.P 7955, Sidi Othmane, Casablanca, Morocco

\section{Abdellatif Maslouhi} \\ Interdisciplinary Laboratory for Natural Resources and Environment, \\ Department of Physics, Faculty of Sciences, Ibn Tofail University, B.P 242, \\ 14000 Kenitra, Morocco

\section{Moumtaz, Razack} \\ Department of Hydrogeology UMR 6532, University of Poitiers, 40 Avenue \\ du Recteur Pineau, 86022 Poitiers Cedex, France
}

doi: 10.19044/esj.2017.v13n12p195 URL:http://dx.doi.org/10.19044/esj.2017.v13n12p195

\begin{abstract}
We study the transient two-dimensional flow problems related to the recharge of groundwater using an approach based on the physics of water transfer in the whole domain, including both the saturated and the unsaturated zones. Such a domain is limited by the soil surface and by the lower impervious boundary of the aquifer. The flow problem is solved using a numerical iterative procedure based on the alternate directions implicit finite differences method. To simulate the water transfer in an unsaturatedsaturated porous medium, we developed a mathematical model based on a single flow equation used for both zones. To this end, the unsaturated and saturated zones are regarded as only one continuum and the Richards equation is used for both zones. The simulated results were validated by the calculation of the mass balance, then on values obtained by an experimental model of $160 \mathrm{~cm}$ length and $50 \mathrm{~cm}$ in height. The numerical model reproduces in a satisfactory way the experimental results giving the spacetime evolution of the level of water table.
\end{abstract}

Keywords: Porous Medium, Unsaturated-Saturated, Numerical Model, Finite Difference, Physical Model, Experimental Model 


\section{1- Introduction}

The transient flow problems through the homogeneousheterogeneous porous media were the subject of many studies (e.g.Abassi et al., 2003). A detailed attention was given to the transfers problems through the unsaturated zone (e.g.Vauclin, 1975). Generally, the models used are based on the resolution of the Richards equation. In this direction, various mathematical and numerical approaches were used for the study of these phenomena, we can quote some works related to this problem, such as the study of the flow through the unsaturated-saturated zone with permanent mode carried out by Vauclin et al., (1979). To solve this problem in unstationary mode, we used a mathematical formulation used by Ackerer et al. for a monodimensional study (e.g. Diaw et al., 2001) and Saleem Ahmed et al., (1993) for the study of the drains based on the finite element method which consists in not regarding the water-table of the groundwater as a separation limit between the two zones, but to present in the same continuum, the saturated zone and the unsaturated zone under unclaimed. This method makes it possible to use only one equation, the water flow equation, established for the transfers of water in partially saturated medium and which has also the remarkable property to be valid in completely saturated mediumMaslouhi et al., (2009). Moreover, this approach has other advantages especially at the numerical level. Indeed, the mathematical formulation used lends itself easily to a numerical modeling integrating the two zones unsaturated and saturated and thus avoiding the presence effect of the capillary fringe.

The objective of this work is to model and simulate the transitory transfers in the unsaturated and saturated zones. We judged the precision and the validity of our model while basing ourselves, on the one hand, on the mass balance which was respected with a very good precision. In the second time, on the experimental data obtained on the small-scale model developed at the laboratory.

The numerical method is based on the alternate directions implicit scheme (ADI). Each iteration is composed alternatively by column and line sweeping by. The method undertakes a sweeping of the discretised domains column by column then line by line, alternatively. The name, method of alternate directions, comes from this procedure. This method offers the advantage of leading to the resolution of linear systems whith matrices tridiagonals bands (e.g.Lichnevski, 2002). The Simulations enabled us to follow the temporal evolution of the water-table in steady state regime, to determine the hydraulic load fields, to simulate the distribution of water volume reaching the groundwater at various times, and finally, to validate our results with experimental data (e.g.Lemacha et al., 2015). 


\section{2- Methods}

\section{1- Position of the problem}

Figure 1 defines the geometry of the studied system. The limits of the field of flow are:

a- A higher horizontal surface (surface of the ground) on a part of which is applied an initial flow q0 causing the infiltration. The infiltration strip will be able to simulate either an irrigation canal, or an artificial storing reservoir.

b- Equidistant trenches of the infiltration strip axiswhere it would be possible to impose a water level limiting the thickness of the groundwater. Each trench can simulate a ditch of drainage or a tank.

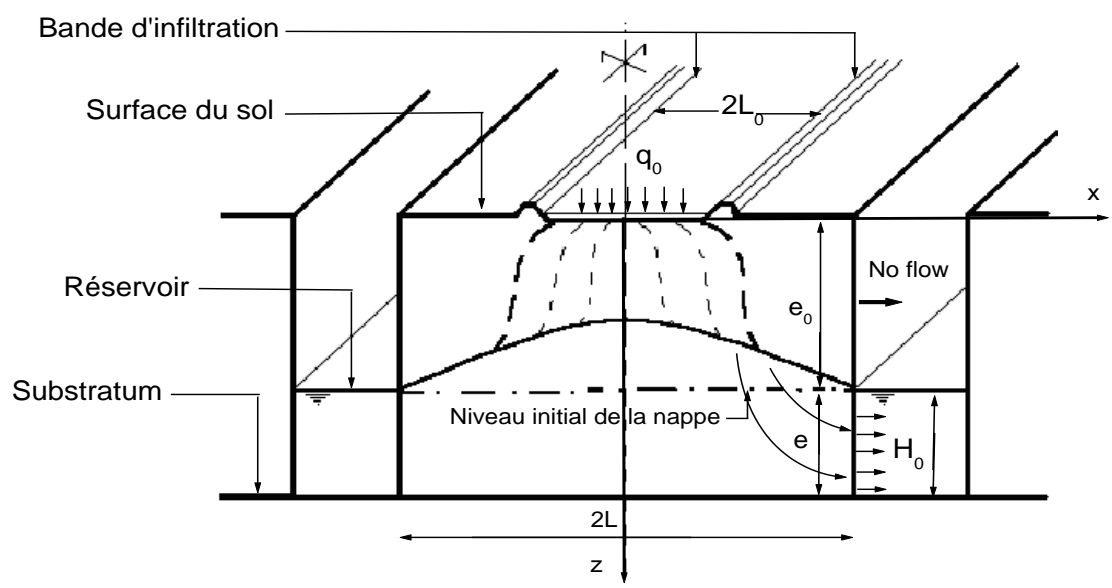

Figure 1: Schematization of the study domain.

q0: Constant infiltration flow (L/T); $\mathrm{e}_{0}$ : Saturated thickness (L);

$\mathrm{H}_{0}$ : Imposed hydraulic head (L); $\mathrm{L}_{0}$ : Width strip (L).

A physical domain which enables to simulate the recharge of a groundwater through the unsaturated zone is considered. This domain (Figure 1) is represented by a groundwater with a horizontal water table, a saturated thickness (e) above an impermeable bottom and at a depth $\left(\mathrm{e}_{0}\right)$

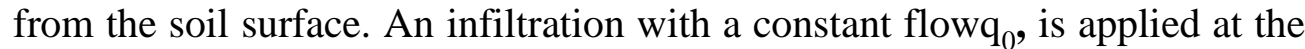
soil surface on a strip of width $2 \mathrm{~L}_{0}$ and of infinite length. The infiltration strip is located between two parallel trenches (regarded as reservoirs) distant of $2 \mathrm{~L}$ from each other andpenetrating to the impermeable layer. A constant piezometric level is maintained in these trenches at the depth $\mathrm{e}_{0}$. 
The plane problem is represented in a system of axes xoz, which is symmetrical according to the axis oz, in this way the study will be reduced to half domain.

\section{2- Basic assumptions}

Taking into account all the phenomena of transfer in the unsaturated zone and the saturated zone with the basement, is very difficult, and even impossible, to realize on the level of modeling. Thus, we find ourselves is in front of several equations with several variables and parameters. This is why some assumptions must be considered in order to specify the field of validity of the equations used:

- The flow is isothermal.

- The liquid phase is the only one moving (neglecting the flow of the gas phase).

- The compression of the gas phase is neglected (the air is always at the atmospheric pressure).

- The porous medium is inert, indeformable, homogeneous, isotropic and to which the law of Darcy is valid.

\section{3- Mathematical model}

Both unsaturated and saturated zones can be regarded as only one continuum. The equation which will be used for both zones has a form close to the Richards equation:

$$
\mathrm{C} \frac{\partial \mathrm{h}}{\partial \mathrm{t}}=\operatorname{div}[\mathrm{K} \operatorname{grad}(\mathrm{h}-\mathrm{z})]
$$

With $\mathrm{h}=$ soil water pressure head $(\mathrm{L}) ; \mathrm{K}=$ hydraulic conductivity $(\mathrm{L} / \mathrm{T}) ; \mathrm{C}=$ coefficient depending on the nature of the zone $\left(\mathrm{L}^{-1}\right)$. Thus the equation (1), by adopting appropriate values of parameters, represents the Richard's equation in the unsaturated zone and the groundwater flow equation in the saturated zone below water-table, for two-dimensional, unstationary flow in a porous media.

Indeed:

- For the unsaturated zone: $(\mathrm{h}<0)$

$\mathrm{C}=\mathrm{C}(\mathrm{h})$

$\mathrm{K}=\mathrm{K}(\mathrm{h})$

$\theta=\theta(\mathrm{h})$

$C(h)=\frac{d \theta}{d h}:$ capillary capacity $\left(L^{-1}\right)$

$\mathrm{K}(\mathrm{h})$ : hydraulic conductivity (L/T) 
$\theta(\mathrm{h}):$ water content $\left(\mathrm{L}^{3} / \mathrm{L}^{3}\right)$

For this zone, we find the traditional Richard's equation applied to the unsaturated zone:

$$
\mathrm{C}(\mathrm{h}) \frac{\partial \mathrm{h}}{\partial \mathrm{t}}=\operatorname{div}[\mathrm{K}(\mathrm{h}) \operatorname{grad}(\mathrm{h}-\mathrm{z})]
$$

- For the saturated zone: $(\mathrm{h} \geq 0)$

$$
\begin{aligned}
& \mathrm{C}=\mathrm{S}_{\mathrm{s}} \\
& \mathrm{K}=\mathrm{K}_{\mathrm{s}} \\
& \theta=\theta_{\mathrm{s}} \\
& \mathrm{S}_{\mathrm{s}}: \text { specific storage coefficient }\left(\mathrm{L}^{-1}\right) \\
& \mathrm{K}_{\mathrm{s}} \text { : saturated hydraulic conductivity }(\mathrm{L} / \mathrm{T}) \\
& \theta_{\mathrm{s}}: \text { saturated water content }\left(\mathrm{L}^{3} / \mathrm{L}^{3}\right)
\end{aligned}
$$

We obtain the diffusivity equation that characterizes the transient flow of water in a saturated porous media, written in a linear form.

$$
S_{s} \frac{\partial h}{\partial t}=\frac{\partial^{2} h}{\partial x^{2}}+\frac{\partial^{2} h}{\partial z^{2}}
$$

Therefore, the formulated problem will be solved taking into account the boundary conditions imposed at the limits of the domain(Figure 2) and the condition imposed at the water table, that is $\mathrm{h}=0$.

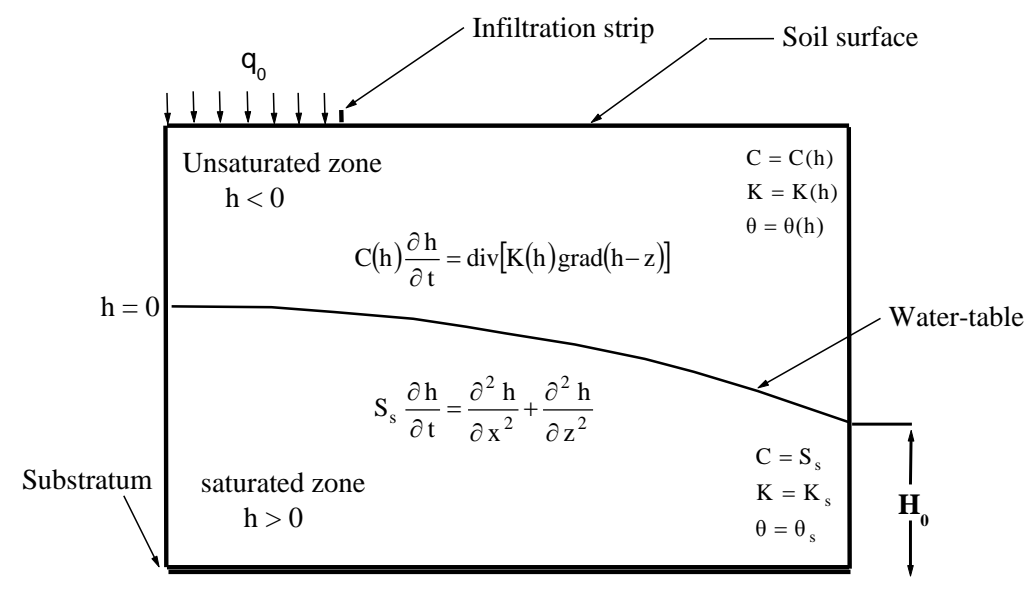

Figure 2: Comprehensive formulation of the recharge problem of a free groundwater.

\section{4- Numerical scheme}


Such a flow problem has actually no analytical solution. The numerical studies carried out on this type of equations relatively very few (e.g. Remson et al., 1971; Freeze, 1969; Taylor, 1970; Verma et al., 1971; Dierch et al, 1999; Ventrella et al., 2000; Diederik et al., 2002). A numerical model was thus developed to search for the hydraulic head distribution at each time step, satisfying the Richard's equation in a porous medium, which $\mathrm{K}(\mathrm{h})$ and $\mathrm{C}(\mathrm{h})$ relations are known in the case of unsaturated zone for defined initial and boundary conditions.

The numerical resolution, which is used, is the Alternating Direction Implicit method (ADI). This method is developed in detail by Peaseman and Rachford,(1955)and Khanji, (1975). It offers the advantage of leading to the resolution of a linear system, having are tri-diagonal bands matrices. It olso reduces the computational time counting from seven to twenty-five times compared to the explicit methods and the Crank-Nicolson's method (e.g.Remson et al., 1971). Moreover, like the latter, it gives a second-order precision in space and time. It is advisable to note however that one should be very carefull while applying ADI method when the domain presents singularities, irregular or returning borders, sources or sinks of flow (e.g.Rushton, 1974).

\section{5- Assembly and experimental unfolding}

For the experimental tests, we developed a research model whose objective is to reproduce at the laboratory the hydraulic of a groundwater drained in controlled fonctioning conditions (figure 3). The model is formed by two symmetrical tanks (e.g.Lemacha, 2006). It is filled with fine sand. To study the follow-up and to visualize the increase of the level of the groundwater due to the infiltration of water starting from the surface of the ground, the model was equiped with a series numbered piezometers throughout one of its edges. To calculate the water level in all the field, a series of five piezometers was placed on the same side line. Given, that one of the interests of the study is to have information relating to the increase of the groundwater level. We have thus recorded the levels of water on the piezometers located at the level of the wall of the model. A rain simulator was installed to feed the field constant flow. With The rain simulator is connected to two pumps with well defined flow (the first pump functions with a flow of one liter by minute and the second one with 0.75 liter by minute) which are located at the bottom of a tank of 220 liters. On the two trenches of the model, tubes of average diameter and a $10 \mathrm{~cm}$ height were placed to preserve a constant load. The quantities of water which run out in the tubes are transferred again to the tank for a new feed back. 


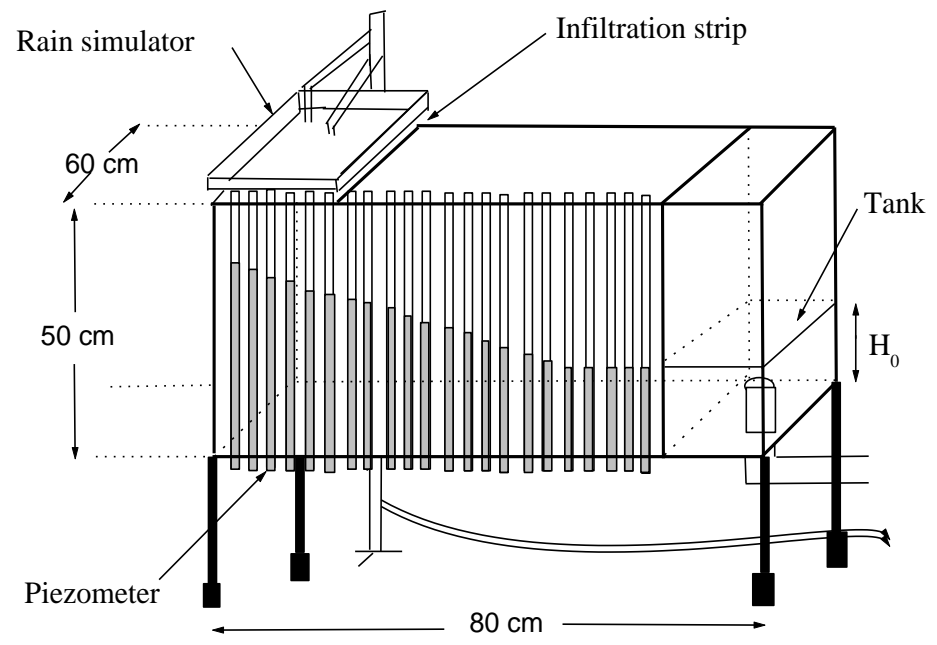

Figure 3: Diagram in prospect for the experimental assembly.

Before starting the experiment, we did too several tests to make sure that the model functions correctly. For that, we raised the level of water in one of the tanks to $40 \mathrm{~cm}$ height and left the level in the other tank to $10 \mathrm{~cm}$, to create a side flow in the model. Concerning the piezometers, we tried to take a good piezometric form of the level which is affected by the appearance of the air bubbles caused by the pressure of ballastings (the elimination of the air bubbles was made by the technique of aspiration all the piezometers). After this stage, we start the first test when the system is in hydrostatic equilibrium. Thanks to a numerical camera fixed at a tripod, we recorded the piezometric levels each 10s. In spite of the precautions which were taken, the increase of the water level in the piezometers is always affected by the air bubbles. To solve this problem, we have remad the tests seven times, the last one gave results which appeared satisfactory to us. We took a hundred photographs until obtaining the permanent mode of the flow, which is obtained at 800 s after the beginning of the refill (when the level of water in the piezometers becomes stable during the refill). The tests showed that the increase of the level of the groundwater under the infiltration strip created a significant hydraulic load, and consequently, the water flow occurs because of the pressure difference between the lower part of the source and the applied $\left(\mathrm{H}_{0}\right)$ by the tranche of the model. On each photograph, we traced the profile of the level of the groundwater. Figure 4 represents the space-time evolution of the profiles of the groundwater level obtained during the test. According to this figure, we observe that the piezometric levels go up in the course of time following the infiltration starting from the unsaturated zone. 
This increase is continuous in time, and stabilized during the last minutes the steady state flow. This mode is explained by the fact that the entering flow emanating from the source becomes equal to that outgoing one by the model discharge system.

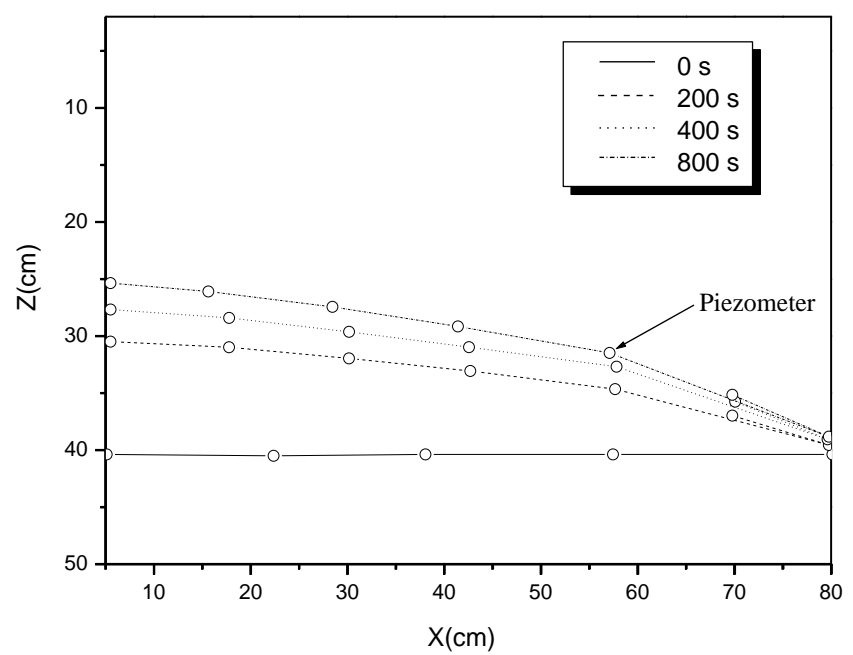

Figure 4: Experimental profiles of the level of the water-table at various moments.

\section{6- Hydrodynamic characterization}

The study of water flow and solute transport in porous media such as soils requires beforehand the determination of their hydrodynamic characteristics. In fact, we should determine the two relations which relate the hydraulic conductivity $\mathrm{K}$ and the water content $\theta$ with the effective pressure head of the soil water $h$. Their determination constitutes an essential step for any study of water flow in the unsaturated zone (e.g. Nayagum et al., 2001; Saâdi et al., 2003; Tamoh et al., 2004). Several experimental techniques can be used to determine them. Their determination in the field presents some difficulties related to several causes, mainly spatial variability concerning the scale parameters, like saturated hydraulic conductivity $\mathrm{K}_{\mathrm{s}}$, the soil heterogeneity, and some errors due to the type of field experiments. Nevertheless, there in situ determination of these parameters is in crucial importance for the study of water transfer in soils.

These relations are however very different according to the type of medium in which the flow occurs.

The $h(\theta)$ relation makes it possible to characterize the flows of water in unsaturated medium. Just like for the $K(\theta)$ relation (figure 5), of many work were realized to determine laws of evolution of the potential of 
pressure according to volumetric moisture. Among the principal relations thus, we note those of Gardner, (1958), Brooks and Corey, (1964) and Van Genuchten, (1980).

\section{7- Hydrodynamic properties of the soil}

The soil parameters used in this work (Table 1), are those of the Mnasra zone in Morocco (soil with sandy loam texture with a sandy tendency). The hydrodynamic characteristics of this soil type are given by Saâdi and Maslouhi, (1998).

$$
\begin{aligned}
& \theta=\theta_{s}\left[1+\left(\frac{h}{h_{g}}\right)^{r}\right]^{-p} \\
& K=K_{s}\left(\frac{\theta}{\theta_{s}}\right)^{\eta}
\end{aligned}
$$

With

$p, r$ and $\eta$, constants
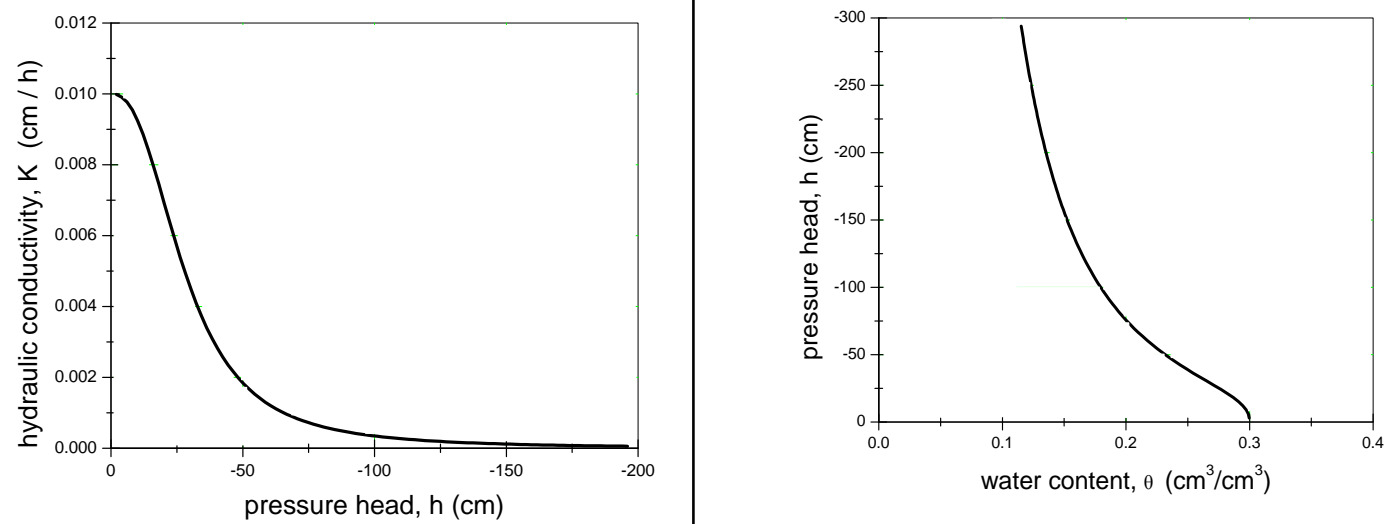

Figure 5: $K(h)$ and $h(\theta)$ used curves.

\section{8- Parameters}

To validate the numerical model, we used on the one hand, our experimental measures which were taken on a model with reduced dimensions and which was filled with fine sand whose hydro-dispersive characteristics are gathered in table 1, and in addition, to some experimental measures coming from the literature (e.g.Khanji, 1975). Table 1 presents the soil parameters values and some data used in our physical model. 
Concerning the numerical simulations, the part of the code, relating to the infiltration, was adapted to the type of test to simulate (constant flow).

Taking into account the soil heterogeneities, there exists on certain driving positions, a light difference between the values of the water contents. Since we suppose in the numerical model that the soil is homogeneous and is characterized by a $\theta(\mathrm{h})$ relation which is the average of the suction curves measured on each vertical.

Table 1- Parameters of the used model.

\begin{tabular}{lc}
\hline Parameters & Values \\
\hline $\mathrm{X}_{\text {max. }}$ & $80 \mathrm{~cm}$ \\
$\mathrm{Z}_{\text {max. }}$ & $50 \mathrm{~cm}$ \\
$\Delta \mathrm{x}$ & $5 \mathrm{~cm}$ \\
$\Delta \mathrm{z}$ & $3 \mathrm{~cm}$ \\
$\mathrm{~S}_{\mathrm{s}}$ & $4.610^{-5} \mathrm{~cm}^{-1}$ \\
$\mathrm{~T}_{\max .}$ & $5600 \mathrm{~s}$ \\
$\mathrm{~K}_{\mathrm{s}}$ & $0.0225 \mathrm{~cm} / \mathrm{s}$ \\
$\mathrm{h}_{\mathrm{g}}$ & $30 \mathrm{~cm}$ \\
$\Delta \mathrm{t}$ & $10 \mathrm{~s}$ \\
$\theta_{\mathrm{s}}$ & $0.3 \mathrm{~cm} \cdot \mathrm{cm}^{-3}$ \\
$\mathrm{q}_{0}$ & $1.94 \mathrm{~cm} / \mathrm{s}$ \\
$\mathrm{H}_{0}$ & $10 \mathrm{~cm}$ \\
$\mathrm{n}$ & 17 \\
$\mathrm{~m}$ & 17 \\
$\eta$ & 6.55 \\
$\mathrm{p}$ & 0.173 \\
$\mathrm{r}$ & $2 /(1-\mathrm{p})$ \\
$\omega$ & 0.38 \\
\hline
\end{tabular}

$\mathrm{X}_{\max }$ :Length of the study domain (L); $\mathrm{Z}_{\max }$ :Depth of the study domain (L); $\mathrm{T}_{\max }$ :Maximum time (T); $\Delta$ t: Time step (T); $\Delta \mathrm{x}$ :horizontal space step (L); $\Delta \mathrm{z}$ : vertical space step (L); $\mathrm{n}$ :Number of horizontal nodes; m: Number of vertical nodes ; $\mathrm{h}_{\mathrm{g}}$ : the scale parameter of water pressure head (L of water); $\omega$ : effective porosity; $p, r$ and $\eta$ : constants.

\section{3- Results and discussion}

\section{1- Mass Balance}

To validate the numerical model, we calculated the mass balance which represents the relationship between variantion of volumes of water entering and outgoing compared to the variation of the water stored in the field. The interest of the calculation of the mass balance is to check the solutions quality obtained by the numerical code for each iteration. According to figure 6 , the curve representing the mass balance is close to 
value 1, except during the first minutes when the flow gradient in the lower part of the infiltration strip is very strong. It does not go down therefore under (0.9). This enables to state that the model satisfies quite well the principle of the mass conservation.

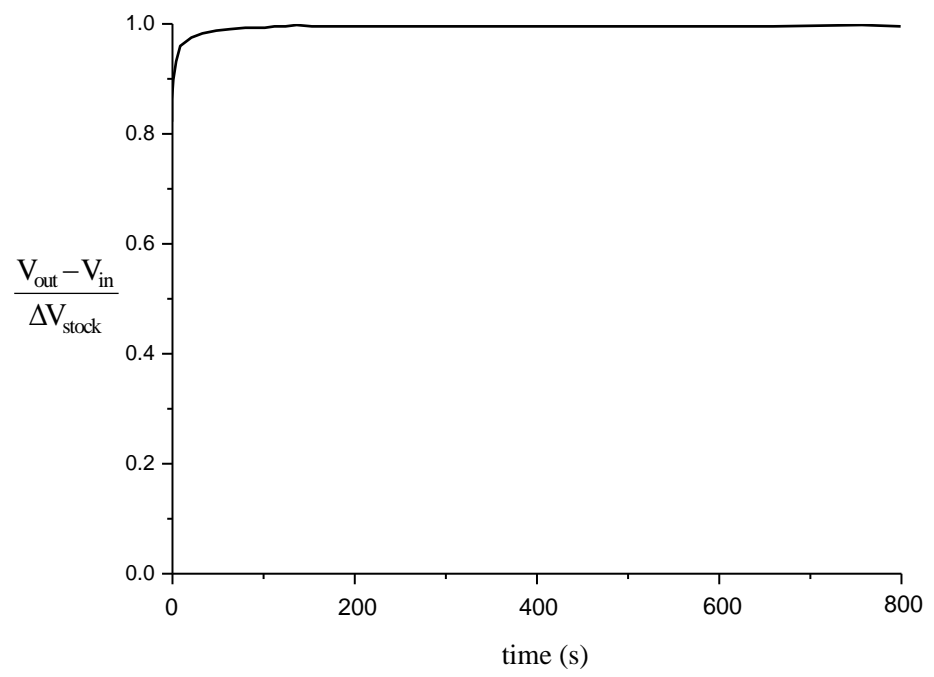

Figure 6: Water mass balance.

\section{2- Space-time evolution of the groundwater level}

In this part, we will use the experimental results obtained to validate the numerical model used. Figure 7 shows the temporal evolution of the water table profiles measured and calculated by the finite difference method (ADI). We note that there is a rising of the water table in time. This rising is due, primarily, to the recharge of the groundwater from the unsaturated zone. We also note that the water table rising still goes on after 400 s and even 800 s. This reflects the flow regime, which is unsteady due to the fact that flow entering the domain by the infiltration strip $\left(\mathrm{V}_{\text {in }}\right)$ is higher than that outgoing through the outlet $\left(\mathrm{V}_{\text {out }}\right)$. This will create a head gradient in the saturated zone between points located beneath the source zone and those close to the fixed head boundary. Figure 7 indicates a good agreement between the positions of measured and calculated water table profiles during all the recharge periods. The position of the water table at $t=800$ s indicates that the flow regime has turned to a steady regime. The comparison between the numerical and experimental results shows that the response of the numerical model developed here is quite satisfactory. Indeed, the model manages to represent correctly the physical processes, if the initial and boundary conditions are expressed on known and fixed in time geometrical limits. It is quite obvious that the numerical results could be improved by 
decreasing the steps of time and space. However, the profit of precision, which would result from it, would be affectedby a more significant cost and would be illusory, taking into account the inaccuracies with which the characteristic relations $\mathrm{K}(\mathrm{h})$ and $\mathrm{h}(\theta)$ are estimated.
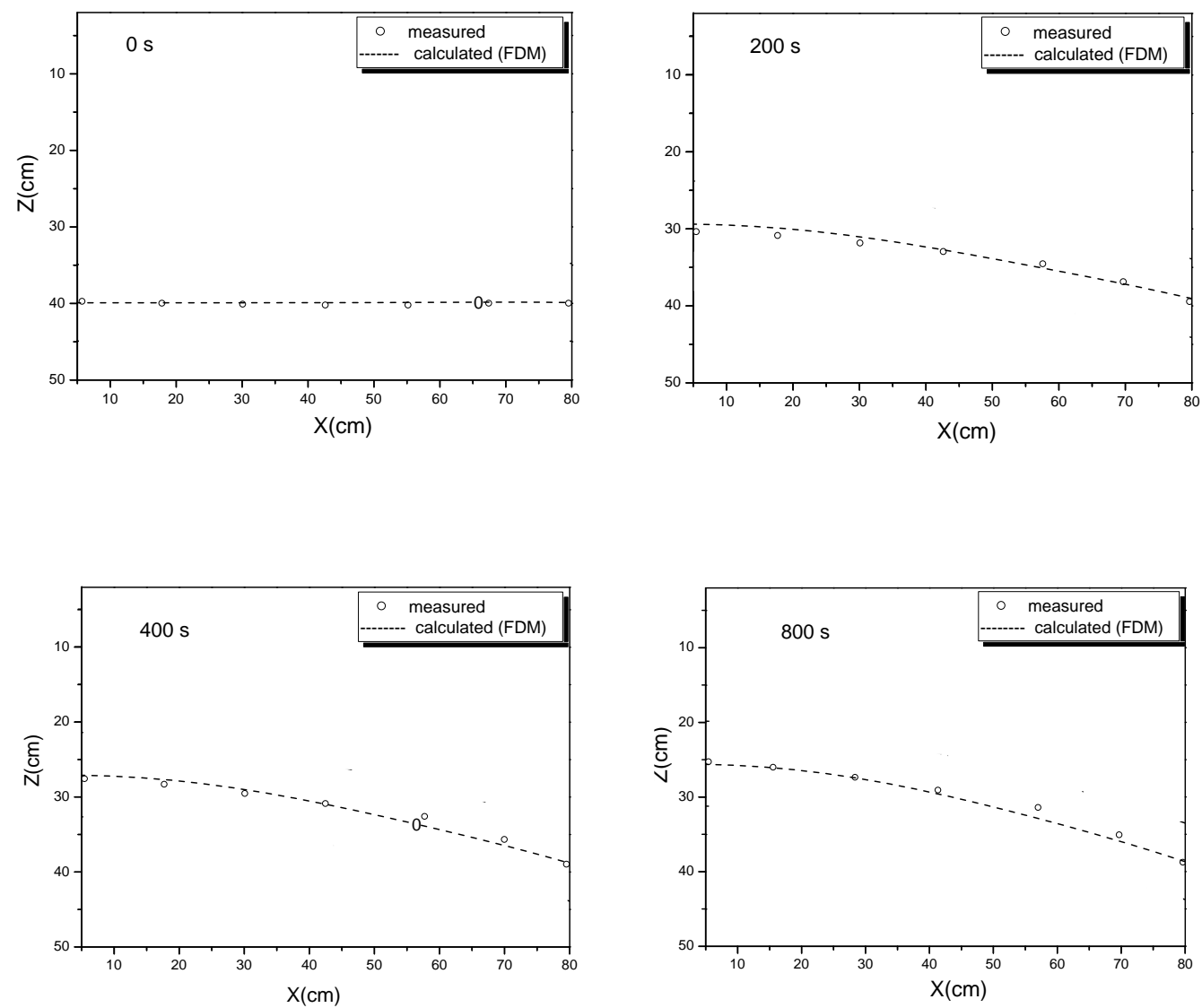

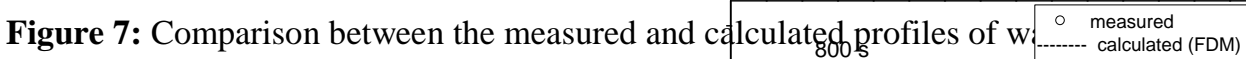
$\mathrm{t}=0,200,400$ arpd $800 \mathrm{~s}$.

In addition, we compared the rescêth e increase of the piezometric level of the groundwater to kheassures-eoming fro aturis obtained for the steady regime (e.g.Khanj]it profiles of the water-table measured $\mathrm{and}_{40} \mathrm{~s} 1 \mathrm{mulated}$ by the 8 repres ........ used, relating to the steady regime at the end of $8 \mathrm{~h}$. One reaches nuuces

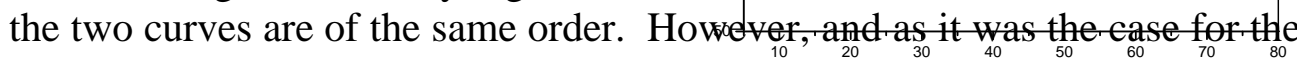


small-scale model, the numerical solution obtained by the numerical code is rather close to the experimental points, except below the infiltration strip.

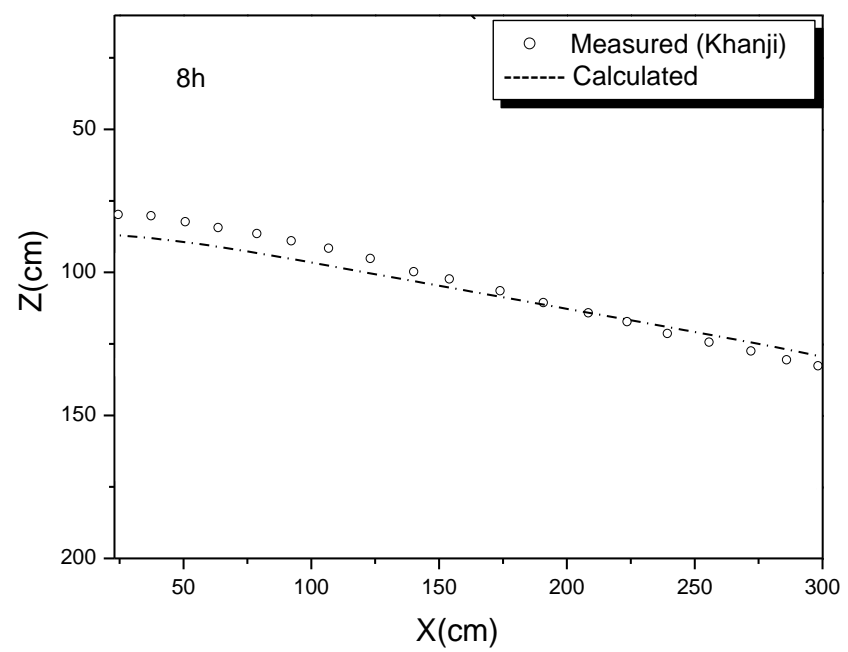

Figure 8: Comparison between the measured (Khanji) and calculated profiles of free face at time $\mathrm{t}=8 \mathrm{~h}$.

The use of the model allowed us finally to obtain informations which are not accessible to measurement, particularly about the flows distribution in the flow domain. Figure 9 shows a plot of the flow distribution at time $\mathrm{t}=$ $300 \mathrm{~s}$, therefore after the beginning of the recharge. At this time, the flows apart from the strip of infiltration are rather weak, although the head gradients are very high. Let us note that the initial head is $H o=-135 \mathrm{~cm}$ in the whole domain. Moreover, there is a significant flow through the water table, with the following characteristic: for $x<125 \mathrm{~cm}$, the flow passing through the water table has a vertical component oriented towards the bottom $\left(\mathrm{q}_{\mathrm{z}}\right.$ positive), whereas for $x>140 \mathrm{~cm}$, the flow vertical components are directed to the top ( $q_{z}$ negative). The unsaturated zone is humidified by the rising of the water table. It is thus worth noting that the water table rising induces a significant flow in the unsaturated zone, with an ascending vertical component, located over the water table. The deformation of the flow lines, with a component which is essentially vertical under the injection zone and essentially horizontal under the water table is also valuable information. 


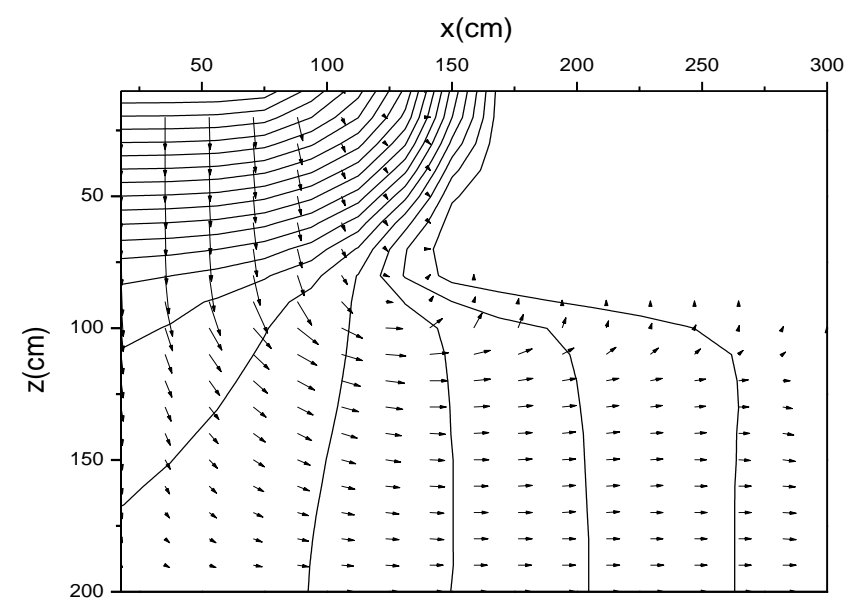

Figure 9: Distribution of hydraulic head calculated at time $t=300$ s.

Figure 10 shows the spatial evolution, after 100s, of the water effective pressure head hfrom the soil surface through the unsaturated zone to the water table which is marked by a pressure $(h=0)$. It is observed that the moisture front (concentric curves of effective pressure iso-values) advances according to time towards the water table surface to supply the groundwater.

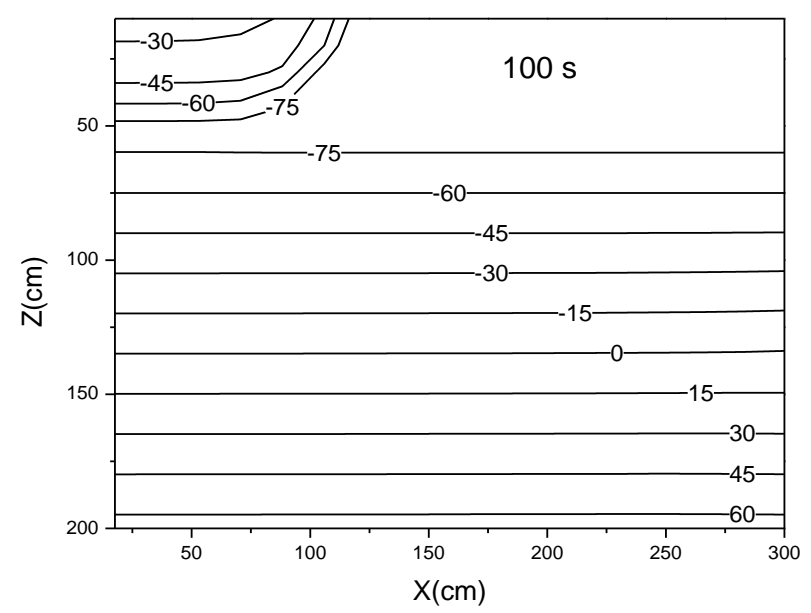

Figure 10: Water pressure head Iso-values after 100s. 
During the refill of the groundwater, we observe that, in the unsaturated zone, the flow entering by the infiltration strip is subdivided in two components, one which horizontal is noted by $\mathrm{q}_{x}$ and a vertical component $\mathrm{q}_{\mathrm{z}}$.According to the results obtained in the first part of our study, we noticed that the vertical component is most dominant, because it is governed by the gravity forces and capillarity. On the other hand, the horizontal component of flow is weak and is due only to the forces of capillarity (phenomena of absorption by capillarity). Concerning the groundwater, we noticed that the horizontal component became most dominant because of the side flow which is produced by a difference in load between the load located at the top of the groundwater and that applied to the discharge system of the groundwater noted by $\mathrm{H}_{0}$. $\mathrm{q}_{\mathrm{z}}$ becomes null.

To try to approach the concept of "effective infiltration" which represents the volume of water reaching the groundwater at a time $t$ compared to the injected volume, let us consider at various times the profile of the flow vertical component passing through a horizontal plane which is located sufficiently close to the groundwater, to be able to suppose that all the flow through this plane reachs the groundwater. Bisthe level of this plane. The profiles shown on figure 11 giving the distribution $q_{z}=f(x)$ through the level $\mathrm{B}$,at three times after the recharge beginning $(t=200 \mathrm{~s}$, 300 s and 800s)as well as the flow profile through a level Acorresponding to the altitude $\mathrm{z}=0$ (injected flow at the soil surface). The integrals $\int q_{z} d x$ provide, at time $t$, the flow per unit soil thickness, passing through the level Band the unit flow considered at the soil surface. The ratio between these integrals directly provides the ratio between the injected fluid and the fluid arriving at the groundwater. 

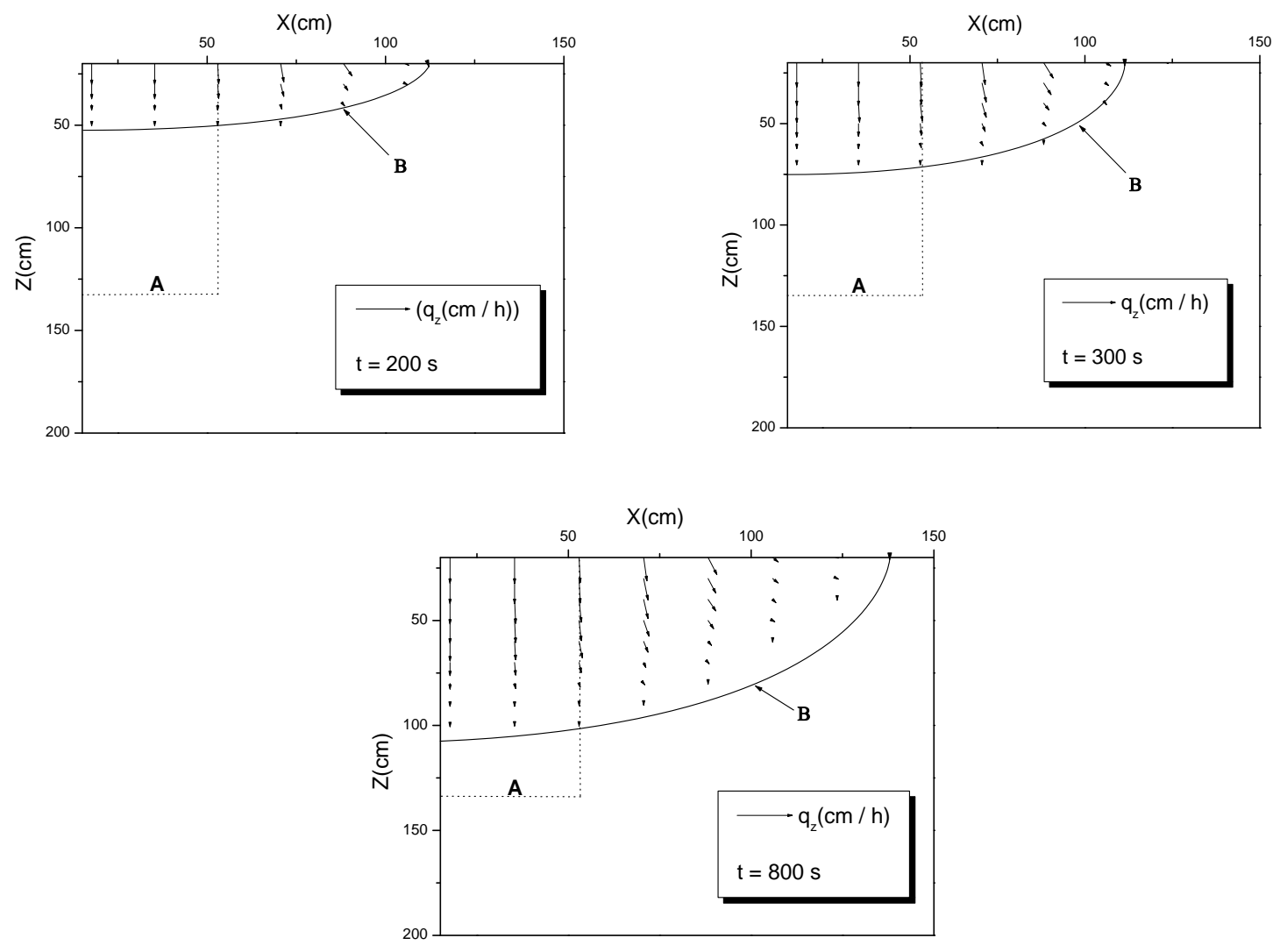

Figure 11: Comparison between the distributions of volumic flow through the soil surface (level A, dotted lines) and arriving at the groundwater (level B, full line) at times $\mathrm{t}=200$, 300 and 800 s.

\section{4- Conclusion}

To model water transfers in the unsaturated zone and the saturated zone of aquifers, we used a mathematical formulation which regards the two zones as only one continuum and a numerical resolution based on numerical scheme ADI. To validate the developed model, we, initially, calculated the mass balance. As a whole, the numerical code answers in a satisfactory way the principle of mass conservation. The results of numerical simulation were also validated on the experimental measures taken on a small-scale model and on results emanating from the literature.

The results presented here were obtained at the laboratory scale. It is essential to envisage their extension to the field scale. The passage to the land parcel scale, then to the catchments scale represents the logical extension to this research. Although, the mathematical model was designed to allow its application to problems of larger space scales, the main 
impediment comes from the difficulty in obtaining, in the field the soil characteristic relations $\mathrm{K}(\mathrm{h})$ and $\theta(\mathrm{h})$ and their strong spatial variability.

Moreover, passing to the field scale often implies the presence of significant heterogeneity, the existence of anisotropy and heat gradients which indisputably restrict, in certain cases, the applications of the results obtained in laboratory. The presence of more or less dense vegetation also modifies the flow transfer and affects the boundary conditions at the soil surface. These various phenomena will be taken into account in coming modeling works based on their physical analysis.

\section{References:}

1. Abassi F., Adamsen D., Hunsaker J., Feyen J., Shouse P., and van Genuchten M Th., (2003). Effects of flow depth on water flow and solute transport in furrow irrigation. Field data analysis. J. Irrig. Drain. Eng., 129(4): 237-246.

2. Brooks R. H. and Corey A. T., (1964). Hydraulic properties of porous media. Hydrol. Pap. 3, Colo. State Univ., Fort Collins, 27 pp.

3. Diaw E.B., Lehmann F. and Ackerer Ph., (2001). One-dimensional simulation of solute transfer in saturated-unsaturated porous media using the discontinuous finite elements method. Journal of Contaminant Hydrology, 51: 197-213.

4. Diederik J., Simunek J., Timmerman A. and Feyen J., (2002). Calibration of Richards' and convection-dispersion equations to field-scale water flow and solute transport under rainfall conditions. Journal of Hydrology, 259: 15-31.

5. Dierch H.-J.G. and Perrochet, P., (1999). On the primary variable switching technique for simulating unsaturated-saturated flows. Adv. Water Resour., 23: 271-301.

6. Freeze R.A., (1969). The mechanism of numerical groundwater recharge and discharge. Water Resour. Res., 5: 153-171.

7. Gardner W. R., (1958). Some steady state solutions of unsaturated moisture flow equation with application to evaporation from a water table. Soil Sci., 85: 228-232.

8. Khanji D., (1975). Etude de la recharge de nappes à surface libre par infiltration. Thèse Doctorat ès Sciences Physiques, Grenoble, France.

9. Lemacha H., (2006). Etude Expérimentale et Numérique des Transferts Hydriques et de Soluté dans un Milieu Poreux non SaturéSaturé. Thèse en Cotutelle, Kenitra, Maroc ; Poitiers, France.

10. Lemacha H., Maslouhi A., Razack M., Mghazli Z. and Hecht F. (2015). Etude expérimentale et modélisation numérique des transferts hydriques et de soluté dans un milieu poreux non saturé-saturé. European Scientific Journal,11, N 15 : 246-259. 
11. Lichnevski A., (2002). Modélisation mathématique: un autre regardIssu de l'épreuve de modélisation de l'agrégation, Scopos, no. 16, Springer.

12. Maslouhi A., Lemacha H. and Razack M. (2009). Modelling of water flow and solute transport in saturated-unsaturated media using a self adapting mesh. IAHS Publ. 331, 2009.

13. Nayagum D., Schafer G. and Mose R., (2001). Approximation par les éléments finis mixtes d'une équation de diffusion non linéaire modélisant un écoulement di-phasique en milieu poreux. C. R., Acad. Sci. Paris. 239: 87-90.

14. Peaseman D.W. and Rachford H.H., (1955). The numerical solution of parabolic and elliptic differential equations. J. Soc. Ind. Appl. Math., 3: 28-41.

15. Remson I., Hornberger G.M. and Molz F.D., (1971). Numerical methods in subsurface hydrology. John wiley, New York.

16. Rushton K.R., (1974). Critical analysis of the alternating direction implicit method of aquifer analysis. J. Hydrology, 2: 153-172.

17. Saadi Z. and Maslouhi A., (1998). Soil vadose zone characterization at large scale for unsaturated water flow and solute transfer modeling, in: international symposium on computer methods for engineering in porous media, Giens, France.

18. Saâdi Z. and Maslouhi A., (2003). Modeling nitrogen dynamics in unsaturated soils for evaluating nitrate contamination of Mnasra groundwater. Advances in Environmental Research, 7: 803-823.

19. Saleem Ah., Deepak K. and Mathur B. S., (1993). Mathematical Modeling of Saturated-Unsaturated Flow to Drains. J. Irrig. Drain. Eng., 119: 18-33.

20. Tamoh K. and Maslouhi A., (2004). Mesures in situ par infiltrométrie des propriétés hydrodynamiques des sols de Mnasra (Maroc). C. R., Acad. Sci., 336: 535-545.

21. Taylor J. L., (1970). Etude expérimentale des phénomènes d'hystérésis dans les écoulements en milieu poreux non saturé. Thèse de docteur ingénieur, Grenoble, France.

22. Van Genuchten M. Th., (1980). A closed-form equation for predicting the hydraulic conductivity of unsaturated soils. Soil Sci. Am. J., 44: 892-898.

23. Vauclin M., (1975). Etude expérimentale et numérique du drainage de nappes à surface libre. Influence de la zone non saturée. Thèse Doctorat ès Sc. Physiques, Grenoble, France.

24. Vauclin M., Khanji D. and Vachaud G., (1979). Experimental and Numerical Study of a Transient, Tow-Dimensional Unsaturated- 
Saturated Water Table Recharge Problem. Water Resour. Res., 5: 1089-1101.

25. Ventrella D., Mohanty B. P., Simunek J., Losavio N. and Van Genuchten M. Th., (2000). Water and chloride transport in a finetextured soil: filed experiments and modeling. Soil Sci., 165: 624631.

26. Verma R.D. and Brutsaert W., (1971). Unsteady free surface groundwater. Proc. American soc. Civ. Engrs, HY8, 1213-1229. 